\title{
Influence de la nature du foin et de l'état alimentaire sur la teneur en matière sèche et la granulométrie des digesta à différents niveaux du réticulo-rumen de la vache
}

\author{
R Baumont, J Jamot, C Dardillat, E Grenet \\ INRA, centre de Clermont-Ferrand-Theix, unité ingestion, \\ station de recherches sur la nutrition des herbivores, \\ 63122 Saint-Genès-Champanelle, France
}

\begin{abstract}
Summary - Influence of the nature of hay and of the alimentary status on the dry matter content and the particle size of digesta at different levels of the reticulo-rumen of the cows. The effect of nature of hay and of alimentary status was studied on the dry matter content and the particle size of digesta at different levels in the reticulo-rumen of the cow. The results support the concept of the filter bed effect of rumen digesta in the sorting out of particles and point out differences among hays.
\end{abstract}

La stratification des digesta dans le réticulo-rumen $(R R)$ dépend des caractéristiques des particules alimentaires (taille, densité...) et conditionne pour partie leur temps de séjour dans cet organe (Evans et al, 1973). Nous avons étudié, avant et après le repas suivant la distribution de foin, l'influence de la nature du foin sur la teneur en matière sèche (MS) et la taille des particules à 3 niveaux dans le rumen (sac dorsal au niveau de la canule, sac ventral juste en dess sus des piliers du rumen et fond du sac ventral) et dans le réseau.

Matériel et méthodes - Trois vaches laitières taries $(735 \pm 15 \mathrm{~kg})$ munies de canules du rumen ont été alimentées ad lib selon un schéma en carré latin $3 \times 3$ avec un foin tardif de prairie naturelle et des foins précoces de dactyle et de luzerne (digestibilité de la MS : 54,66 et $64 \%$; niveau d'ingestion : $8,6,10,9$ et $11,1 \mathrm{~kg}$ de MS respectivement). Les digesta ont été échantillonnés juste avant les distributions de foin ( 9 et $21 \mathrm{~h}$ ) et $2 \mathrm{~h}$ après : en premier, ceux du fond du sac ventral et du réseau, par aspiration; puis ceux du sac dorsal et du sac ventral au cours du vidage du RR. La granulométrie des échantillons a été déterminée par tamisage par voie humide (Grenet, 1970).

Résultats et discussion - La teneur en MS du contenu du sac dorsal (tableau I) est généralement significativement supérieure à celle du contenu du sac ventral. La granulométrie du contenu du sac ventral a tendance à être plus fine que celle du sac dorsal, les différences maximales étant observées pour la luzerne après le repas. Dans les sacs dorsaux et ventraux, la proportion de particules de taille $>2 \mathrm{~mm}$ est significativement plus faible pour le foin de prairie que pour les 2 autres foins. Le foin de dactyle se distingue des 2 autres par une abondance des très fines particules $(<0,25 \mathrm{~mm})$ aux dépens des particules de taille moyenne $(0,25$ à $2 \mathrm{~mm})$.

Les contenus du fond du sac ventral (FSV) et du réseau ont une teneur en MS nettement plus faible que celle du 
Tableau I. Influence de la nature du foin et de l'état alimentaire sur la teneur en matière sèche (MS) et la répartition des particules (en \% de la MS retenue) dans le réticulo-rumen chez 3 vaches $(n=18)$.

\begin{tabular}{|c|c|c|c|c|c|c|c|}
\hline & Prairie $r$ & aturelle & Dac & $y l e$ & Luze & rne & \\
\hline & $\begin{array}{l}\text { Avant } \\
\text { repas }\end{array}$ & $\begin{array}{l}\text { Après } \\
\text { repas }\end{array}$ & $\begin{array}{l}\text { Avant } \\
\text { repas }\end{array}$ & $\begin{array}{l}\text { Après } \\
\text { repas }\end{array}$ & $\begin{array}{l}\text { Avant } \\
\text { repas }\end{array}$ & $\begin{array}{l}\text { Après } \\
\text { repas }\end{array}$ & PPDS \\
\hline $\begin{array}{l}\text { Sac c'orsal } \\
\text { Teneur en MS (\%) } \\
>2 \mathrm{~mm} \\
0,25 \text { à } 2 \mathrm{~mm} \\
<0,25 \mathrm{~mm}\end{array}$ & $\begin{array}{l}12,3 \\
26,9 \\
51,3 \\
21,8\end{array}$ & $\begin{array}{l}13,3 \\
37,5 \\
43,7 \\
18,9\end{array}$ & $\begin{array}{l}11,7 \\
38,1 \\
27,6 \\
34,3\end{array}$ & $\begin{array}{l}13,0 \\
47,5 \\
22,8 \\
29,8\end{array}$ & $\begin{array}{l}12,3 \\
38,3 \\
43,1 \\
18,6\end{array}$ & $\begin{array}{l}13,7 \\
46,6 \\
36,6 \\
16,9\end{array}$ & $\begin{array}{l}1,3 \\
5,9 \\
4,6 \\
4,5\end{array}$ \\
\hline $\begin{array}{l}\text { Sac ventral } \\
\text { Teneur en MS (\%) } \\
>2 \mathrm{~mm} \\
0,25 \text { à } 2 \mathrm{~mm} \\
<0,25 \mathrm{~mm}\end{array}$ & $\begin{array}{l}10,5^{*} \\
24,6 \\
51,6 \\
23,9\end{array}$ & $\begin{array}{l}11,8 \\
36,0 \\
44,6 \\
19,4\end{array}$ & $\begin{array}{l}10,7 \\
35,5 \\
29,1 \\
35,5\end{array}$ & $\begin{array}{l}10,7^{*} \\
44,9 \\
26,4 \\
28,7\end{array}$ & $\begin{array}{l}11,1^{*} \\
34,1 \\
44,7 \\
21,2\end{array}$ & $\begin{array}{l}11,8^{*} \\
40,9^{\star} \\
40,6^{*} \\
18,4\end{array}$ & $\begin{array}{l}1,5 \\
7,9 \\
5,6 \\
3,8\end{array}$ \\
\hline $\begin{array}{l}\text { Fond du sac ventral } \\
\text { Teneur en MS (\%) } \\
>2 \mathrm{~mm} \\
0,25 \text { a } 2 \mathrm{~mm} \\
<0,25 \mathrm{~mm}\end{array}$ & $\begin{array}{r}4,7 \\
13,5 \\
39,9 \\
46,7\end{array}$ & $\begin{array}{r}3,0 \\
15,3 \\
33,5 \\
51,2\end{array}$ & $\begin{array}{r}4,3 \\
15,6 \\
18,9 \\
65,5\end{array}$ & $\begin{array}{r}3,7 \\
16,2 \\
14,1 \\
69,8\end{array}$ & $\begin{array}{r}6,0 \\
14,3 \\
36,8 \\
48,9\end{array}$ & $\begin{array}{r}4,7 \\
24,7 \\
35,1 \\
40,2\end{array}$ & $\begin{array}{r}1,2 \\
7,7 \\
7,8 \\
10,7\end{array}$ \\
\hline $\begin{array}{l}\text { Réseau } \\
\text { Teneur en MS (\%) } \\
>2 \mathrm{~mm} \\
0,25 \text { a } 2 \mathrm{~mm} \\
<0,25 \mathrm{~mm}\end{array}$ & $\begin{array}{c}5,6 \\
18,8 \\
46,2^{* \star} \\
35,9^{* *}\end{array}$ & $\begin{array}{l}4,7 \\
20,7 \\
41,0^{* *} \\
38,3\end{array}$ & $\begin{array}{l}5,6^{\star \star *} \\
23,3^{\star *} \\
22,9 \\
53,9\end{array}$ & $\begin{array}{l}4,2^{\star *} \\
25,1^{* *} \\
18,3 \\
56,6\end{array}$ & $\begin{array}{c}6,5 \\
23,2^{\star \star} \\
40,0 \\
36,8\end{array}$ & $\begin{array}{r}5,3 \\
24,8 \\
35,8 \\
39,4\end{array}$ & $\begin{array}{r}0,9 \\
9,0 \\
7,0 \\
11,7\end{array}$ \\
\hline
\end{tabular}

PPDS : plus petite différence significative; " différence significative $(P<0,05)$ entre le sac dorsal et le sac ventral; "* idem pour le fond du sac ventral ef le réseau.

contenu des sacs dorsaux et ventraux ainsi qu'une granulométrie beaucoup plus fine. Le contenu du FSV qui rejoint ensuite le réseau pour être évacué par l'orifise réticulo-omasal (Waghorn et Reid, 1977) est donc bien le résultat d'une autofiltration du contenu ruminal (Welch, 1982). Sauf pour la luzerne après le repas, la granulométrie du FSV est plus fine que celle du réseau qui reçoit les particules ingérées et ruminées. Par rapport au foin de prairie tardif, la proportion plus élevée de particules de taille $<0,25 \mathrm{~mm}$ dans le FSV et le réseau avec le dactyle et la teneur en MS plus élevée avec la luzerne peuvent expliquer le transit plus rapide observé avec ces 2 foins (Baumont, 1989). Après le repas, la teneur en MS et la proportion de grandes par- ticules (> $2 \mathrm{~mm}$ ) sont augmentées dans les sacs dorsaux et ventraux alors que la teneur en MS est diminuée dans le FSV et le réseau.

En conclusion, nos résultats mettent en évidence la stratification des digesta dans le $R R$ qui est le résultat d'une autofiltration du contenu ruminal et qui varie avec la nature du foin et l'état alimentaire.

Baumont $R$ (1989). Thèse de doctorat, INA$P G, 159 p$

Evans EW, Pearce GR, Burnett J, Pillinger $S L$ (1973) $B r J$ Nutr 29, 357-376

Grenet E (1970) Ann Biol Anim Biochim Biophys 10, 643-65

Waghorn GC, Reid CSW (1977) Proc NZ Soc Anim Prod 37, 176-181

Welch JG (1982) J Anim Sci 54, 885-894 